\title{
Preface to the Special Issue on user modeling for personalized interaction with music
}

\author{
Marko Tkalčič $^{1} \cdot$ Markus Schedl $^{2} \cdot$ Peter Knees $^{3}$
}

Published online: 24 April 2020

(c) Springer Nature B.V. 2020

\section{Introduction}

Music search, retrieval, and recommendation systems have experienced a boom during the past few years due to streaming services providing access to huge catalogs anywhere and anytime. These streaming services collect the user behavior in terms of actions on music items, such as play, skip, playlist creation, and modification. As a result, an abundance of user and usage data has been collected and is available to companies and academics, allowing for user profiling and to create personalized music search and recommendation systems. The importance and timeliness of research on such personalized music systems is evidenced by publications in venues including the ACM Conference on Recommender Systems, ACM Conference on User Modeling, Adaptation and Personalization, International Society for Music Information Retrieval Conference, the ACM Special Interest Group on Information Retrieval Conference, ACM CHI Conference on Human Factors in Computing Systems, and the ACM International Conference on Intelligent User Interfaces, as well as in journals including IEEE Transactions on Affective Computing and ACM Transactions on Intelligent Systems and Technology, in addition to UMUAI. On the other hand, there are still plenty of unsolved challenges. In particular, scholars have identified as some of the most vital ones: understanding and modeling users, personalization of recommendation and retrieval systems, user adaptivity in interfaces, and context awareness.

Markus Schedl

markus.schedl@jku.at

Marko Tkalčič

marko.tkalcic@gmail.com

Peter Knees

peter.knees@tuwien.ac.at

1 University of Primorska, Glagoljaska 8, Koper, Slovenia

2 Johannes Kepler University Linz (JKU) and Linz Institute of Technology (LIT), Altenberger Straße 69, 4040 Linz, Austria

3 TU Wien, Favoritenstraße 9-11/194-1, 1040 Vienna, Austria 


\section{Papers in the Special Issue}

This special issue contains articles that contribute to the state of the art on (a) personalized user interfaces for music (Jin et al. 2019; Narducci et al. 2019) and (b) playlist creation (Kamehkhosh et al. 2019).

The paper Effects of personal characteristics in control-oriented user interfaces for music recommender systems (Jin et al. 2019) deals with the personalization of control elements in user interfaces for music recommender systems. The authors explore personal characteristics and the user interface characteristics (controls, visualizations, and complexity) to identify the interplay of these variables on the perception of recommendations. In summary, they found that the user characteristic musical sophistication influences the acceptance of the recommendations.

The paper An investigation on the user interaction modes of conversational recommender systems for the music domain (Narducci et al. 2019) addresses the issue of how to design conversational recommender systems for music. The authors evaluated several approaches to the design of a conversational user interface and concluded, based on a user study, that the highest user acceptance of such an interface is when a mixed approach is used, i.e., allowing the user to interact both with natural language and buttons.

The paper Effects of recommendations on the playlist creation behavior of users (Kamehkhosh et al. 2019) investigates if and how recommendations influence the manual playlist creation process. The results of the authors' user study indicate that the users indeed do use recommendations for playlist creation. However, they also identified a delicate balance, i.e., if the recommendations were too diverse, users tended not to use the recommended items in their playlists.

\section{Outlook}

The papers in this special issue address specific challenges related to the user interaction and the influence of the recommendations on user behavior. While the included articles substantially contribute to the state of the art, they open even more research avenues.

A common theme in the special issue is that in music personalization, there are a lot of factors that influence the user experience. Hence, it is of utter importance to design the experiments in such a way that only the observed variables are isolated.

A related issue is also the lack of datasets, so the researchers needed to carry out expensive user studies. Although datasets are not always alternatives to user studies, especially if the research questions deal with unexplored features, it would be beneficial to have at disposal feature-rich datasets that would allow comparisons between similar studies.

Both the papers Narducci et al. (2019) and Kamehkhosh et al. (2019) show the importance of the user intent, which has been neglected in the past for the more 
accessible variables of context. Other user-centric variables, such as affect and personality, have also been identified in related work as important and will likely be up-and-coming topics in future research.

\section{References}

Jin, Y., Tintarev, N., Htun, N.N., Verbert, K.: Effects of personal characteristics in control-oriented user interfaces for music recommender systems. User Model. User-Adapt. Interact. (2019). https://doi. org/10.1007/s11257-019-09247-2

Kamehkhosh, I., Bonnin, G., Jannach, D.: Effects of recommendations on the playlist creation behavior of users. User Model. User-Adapt. Interact. (2019). https://doi.org/10.1007/s11257-019-09237-4

Narducci, F., Basile, P., de Gemmis, M., Lops, P., Semeraro, G.: An investigation on the user interaction modes of conversational recommender systems for the music domain. User Model. User-Adapt. Interact. (2019). https://doi.org/10.1007/s11257-019-09250-7

Publisher's Note Springer Nature remains neutral with regard to jurisdictional claims in published maps and institutional affiliations.

Marko Tkalcic is associate professor at the Faculty of Mathematics, Natural Sciences and Information Technologies (FAMNIT) at the University of Primorska in Koper, Slovenia. He aims at improving personalized services (e.g. recommender systems) through the usage of psychological models in personalization algorithms. Marko has published in journals, such as Springer UMUAI, IEEE Transactions on Affective Computing and at venues, such as ACM IUI, ACM RecSys, and ACM UMAP. He co-edited two books with Springer, "Emotions and Personality in Personalized Services" in 2016 and "Group Recommender Systems" in 2018. He is member of the editorial board of the Springer UMUAI journal. Marko has also co-edited several special issues, most recently the "Psychological Models for Personalized Human-Computer Interaction(HCI)" in Frontiers in Psychology.

Markus Schedl is full professor at the Johannes Kepler University Linz (JKU), affiliated with the Institute of Computational Perception, leading the Multimedia Mining and Search group. In addition, he is head of the Human-centered AI group at the Linz Institute of Technology (LIT) AI Lab. He graduated in Computer Science from the Vienna University of Technology and earned his Ph.D. in Computer Science from the Johannes Kepler University Linz. Markus further studied International Business Administration at the Vienna University of Economics and Business Administration as well as at the Handelshögskolan of the University of Gothenburg, which led to a Master's degree. His main research interests include web and social media mining, data analytics, information retrieval, recommender systems, multimedia, and music information research. He (co-)authored more than 200 refereed conference papers and journal articles, among others, published in ACM Multimedia, RecSys, ICMR, SIGIR, ECIR, ISMIR, IEEE Visualization; Journal of Machine Learning Research, ACM Transactions on Information Systems, IEEE Transactions on Affective Computing, IEEE Multimedia, User Modeling and User-Adapted Interaction (UMUAI), and PLOS ONE.

Peter Knees is Assistant Professor of the Faculty of Informatics of TU Wien, Austria. For over one and a half decades he has been an active member of the music information retrieval community, reaching out to the related areas of multimedia, recommender systems, and text IR. Apart from serving on the program committees of major conferences in these fields, he has organized the RecSys Challenge 2019 as well as several workshops on topics of media retrieval. He is an experienced teacher of graduate-level courses on recommender systems, information retrieval, and data science and has lectured in several tutorials and summer school courses on music information retrieval and recommendation. He co-authored the book "Music Similarity and Retrieval: An Introduction to Audio- and Web-based Strategies" published in the Springer Information Retrieval series and contributed to the chapter on "Music Recommender Systems" in the Recommender Systems Handbook (2nd and 3rd ed.), also published by Springer. Peter is also 
interested in the digital arts and an active promoter of digital humanism, which describes, analyzes, and, most importantly, influences the complex interplay of technology and humankind, for a better society and life, fully respecting universal human rights. 